Chapter 10

\title{
Nanoflora - How Nanotechnology Enhanced the Use of Active Phytochemicals
}

\author{
Fadwa Odeh, Hala Al-Jaber and Dima Khater \\ Additional information is available at the end of the chapter \\ http://dx.doi.org/10.5772/58704
}

\section{Introduction}

Nanotechnology is the science of manipulating matter where at least one dimension is in the nano-scale. Nanotechnology is a very promising field to the medicinal and pharmaceutical sectors, hence, it plays an important role in enhancing humanity's quality of life. Nanotechnology has many applications in the medical sector, including diagnosis and therapeutic. However, therapeutic uses are considered the main application of nanotechnology in the health sector. Nanotechnology has an important impact on improving therapeutics by facilitating drug delivery, increasing the efficacy of the drug, improving its circulation and stability in addition to decreasing its toxic side effects [1]. Most active compounds that are proven to have certain effects to treat certain ailments or alleviate pain can be categorized as organic compounds. Most active organic compounds are insoluble in aqueous media, have poor bioavailability, instable and most of the time are toxic.

Herbal medicines, or active compounds derived from natural sources suffer from the same limitations of many other drugs [2]. However, in the case of synthesized drugs, many of these problems can be overcome by preparing derivatives of these drugs that retain some of their activities and at the same time enhancing their physical properties to a more suitable form for pharmaceutical formulations. However, herbal medicines are still the main source of drugs and their side effects are much lower than their synthetic counterparts, add to this a deep and strongly rooted trust in many societies in their efficacy to heal or prevent diseases, even though most of the time not proven in a scientific and systematic way. In order to overcome such limitations, many techniques have been employed such as solubilization in a non-polar solvent, preparing them as an injection and/or converting the active ingredients into their salt form in order to enhance their solubility in aqueous medium. However, these methodologies also suffer from various disadvantages. These include the toxicity of the solubilizing agent, 
the actual or real activity of the salt form, the need for further studies to insure the presence of the bioactive form of the drug and the extent of its bioavailability. In this aspect, nanotechnology is a very promising tool for enhancing the use of herbal medicines, or in more accurate words, to re-discover their full potential in pharmaceutical formulation. Extensive libraries of nanoparticles-that can be used for the delivery of natural bioactive compounds to a certain target-have been studied. These different nanoparticles can be designed, prepared in different shapes, sizes, compositions, functionalized and modified chemically/physically to suite specific properties depending on the characteristics of both the drug and the targeted organ. These nanoparticles can be anything from emulsion and micro-emulsions, dendrimers, fullerenes, liquid crystals, quantum dots, nano rods, solid lipid nanoparticles (SLN), liposomes, gels and many other different types.

In this chapter, a brief focus on herbal medicines, nanotechnological approaches to enhance their promised action will be reviewed and discussed. At the end, successful stories of nanoparticle loaded active phytochemicals that reached the market will be presented as case studies in this field.

\section{Herbal medicine}

Natural products chemistry is one of the oldest sciences sought for medicine throughout the history of mankind. Its roots dates back in time, thousands of years ago, through the use of many herbal mixtures as remedies for many diseases. It was clear from the beginning that certain herbs, plants, etc... have certain positive influence when used as remedies for sickness, but it was unclear how the mechanism of remedy was attained. As a consequence of the accumulation of many years of knowledge, traditional medicine has come forth to group, organize and categorize these remedies according to their effects on diseases, whereby, biomedicine and chemistry came to shed more light on the active ingredients of these folk remedies and on the mechanism of their action.

Currently, natural product chemistry has evolved to be an interdisciplinary area of science, concerned with the isolation, characterization and determination of the biological activity of the pure phytochemicals. These active components, generally referred to as secondary metabolites, include phenolics, terpenoids, alkaloids and steroids. Even though it has been proved that many natural products have a strong therapeutic value, limitations related to their poor solubility and bioavailability in addition to toxicity and stability have severely hindered their use as drugs [3]. According to U.S. Food and Drug Administration (FDA), the definition of bioavailability is "the rate and extent to which the active ingredient or active moiety is absorbed from a drug product and becomes available at the site of action" [4].

\section{Phenolic compounds}

Natural phenolic compounds are secondary metabolites present in all vascular plants and embracing a vast range of aromatic organic compounds with one or more hydroxyl substitu- 
ent(s). The parent compound is phenol but most of these naturally occurring compounds are polyphenolics, which to date, exceeds 8000 structurally identified compounds. Plant polyphenolics are grouped into different classes depending on their chemical structure. Flavanoids are the largest and most important group of natural polyphenolics with more than 6000 molecules identified so far. Other classes include phenolic quinones, lignans, xanthones, coumarines, polymeric lignins and tanins [5]. Within each class of compounds, the variations around the basic chemical skeleton essentially concern the degrees of oxidation, hydroxylation, methylation, glycosylation and the possible connections to other molecules (primary metabolites such as carbohydrates, lipids, proteins, or phenolic secondary metabolites [6].

Polyphenols were the focus of special attention as a result of the so-called Mediterranean diet rich in fruits and vegetables. This diet appears to protect against cardiovascular diseases in addition to potential beneficial health properties, as evidenced by several epidemiological studies showing that diets rich in fruits and vegetables are generally associated with a lower cancer incidence and other diseases, such as inflammatory or cardiovascular pathologies [7].

The bioavailability of the orally administrated polyphenols is very low due to their low water solubility, poor absorption, extensive and rapid metabolism. To overcome these problems, several bioactive polyphenols were formulated into various pharmaceutical formulations that could improve their bioavailability.

Among the types of naturally occurring polyphenols, flavonoids (Figure 1) are a large and a highly diverse group of structurally related secondary metabolites produced by plants. The main flavonoids subclasses include flavones (Figure 1a), flavonols (Figure 1b), flavans (Figure 1c), flavanones (Figure 1d) in addition to dihidroflavanols (Figure 1e), isoflavons (Figure 1f) and biflavones. [8].<smiles>O=c1cc(-c2ccccc2)oc2ccccc12</smiles><smiles>O=c1c(O)c(-c2ccccc2)oc2ccccc12</smiles>

b<smiles>c1ccc(C2CCc3ccccc3O2)cc1</smiles><smiles>O=C1CC(c2ccccc2)Oc2ccccc2C1=O</smiles><smiles>O=c1c(-c2ccccc2)coc2ccccc12</smiles>

$f$

Figure 1. Basic structures of some flavonoids' backbone

Flavonoids received considerable attention due to their wide biological activities. Many of them are known to possess hepatotoxic, anti-inflammatory, antimicrobial, antiviral, anti- 
allergic and anti-ulcer effects. Also, flavonoids are known to be potent antioxidants with free radical scavenging abilities $[9,10]$. Some flavonoids are known to provide protection against cardiovascular mortality. Moreover, they have been shown to inhibit the growth of various cancer cell lines in vitro, and reduce tumor development in experimental animals [11]. Flavanoids, as natural compounds have several great advantages over therapeutic agents because many diets are rich in flavonoids and polyphenolic compounds [9]. The therapeutic potential of flavonoids makes them valuable targets for drug design [8].

Lignans (Figure 2) are among the important polyphenolic compounds that are recognized with a wide spectrum of biological activities. These compounds generally represent a group of dimeric phenylpropanoids where two C6-C3 are attached by its central carbon C-8.

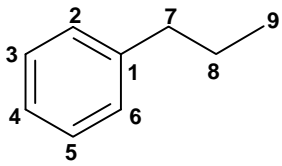

a

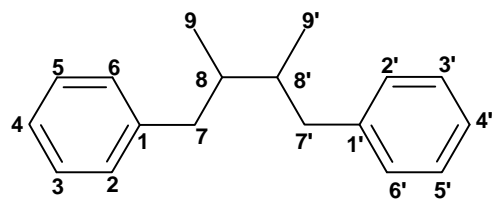

b

Figure 2. Basic structure of lignans: a) Phenylpropanoid unit; b) Lignan structure

Lignans are known for their antiviral, anticancer, cancer prevention, anti-inflammatory, antimicrobial and antioxidant activities. Also, lignans are known for their immunosuppressive, hepatoprotective and osterporosis prevention effects. Podophyllotoxin (Figure 3) has long been known to possess anti-mitotic activity with early clinical trials indicating its high efficacy. Unfortunately, the toxicity of this compound limits its direct application as a drug [12]. To solve the problem of toxicity, several modifications were made to the podophyllotoxin structure in hope of obtaining compounds with low toxicity but retain the desired activities of the parent compound. Thus, etoposide, teniposide, etopophos and GL-331 were synthesized; all are potent chemotherapeutic agents for a variety of tumors. Still, etoposide and its analogues suffer from poor solubility problems.<smiles>COc1cc([C@H]2c3cc4c(cc3[C@@H](O)[C@@H]3COC(=O)[C@H]23)OCO4)cc(OC)c1OC</smiles>

Figure 3. Structure of podophyllotoxin 


\section{Terpenoids}

Terpenoids, as represented by more than 40000 identified compounds, are among the most widespread group of natural products, with several new compounds being discovered every year. Terpenes can be generally defined as a group of molecules whose structure is based on various but definite number of isoprene (3-methyl-1,3-butadiene) units. Based on the number of isoprene building blocks, terpenoids can be classified into monoterpenes (such as thymoquinone), sesquiterpenes, diterpenes (such as retinol, trans-retonic acid, sclareol), sesterterpenes, triterpenes (such as oleanolic and usrolic acids) and tetraterpenes.

From a chemical point of view, terpenoids are usually cyclic unsaturated hydrocarbons, with different degrees of oxygen in the constituent groups attached to the basic isoprene skeleton. A wide range of terpenoids have been found to possess preventive and pharmacological activity against many human ailments including cancer and alzheimer [13, 14]. Several studies also indicated that terpenoids have antimicrobial, antifungal, antiparasitic, antiviral, antiallergenic, anti-spasmodic, antihyperglycemic, anti-inflammatory and immunomodulatory properties [15].

Monoterpenes are among the best known plant secondary metabolites and are one of the main classes of terpenoids detected in essential oils, floral scents and defensive resins (both constitutive and induced) of aromatic plants [16]. A number of monoterpenes have shown antitumor activity. Examples include thymoquinone (Figure 4), limonene and perilla alcohol. Thymoquinone, which is the main active constituent of the essential oil obtained from the medicinal plant Nigella sativa (commonly referred to as Black seeds in Arabic countries), has interesting anticancer, anti-oxidant and anti-inflammatory activities both in vivo and in vitro $[14,17,18,19]$ as well as chemo-preventive properties. The interesting in vitro anticancer activity of this monoterpene against different types of cancer cell lines including human colorectal cancer cells [13], myeloblastic leukemia cells [20], prostate cancer [17] pancreatic adenocarcinoma [14], ovarian and breast adenocarcinoma [21] were faced by several limitations that not only hindered its pharmaceutical applications, but also limited the available suitable approaches that can be used to enhance its bioavailability. Such limitations included the poor solubility, extreme lipophiliciy causing poor formulation characteristics in addition to light and heat instability.
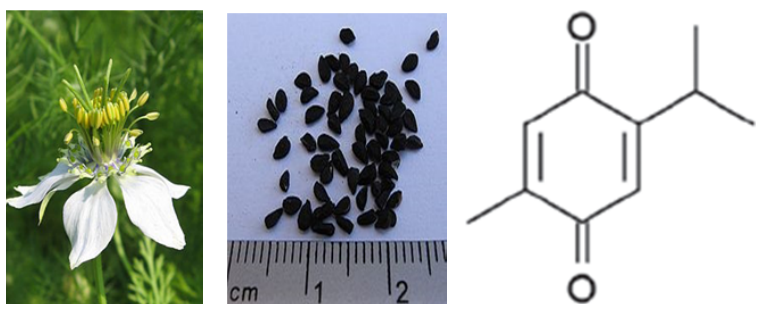

Figure 4. Flower and seeds of N.sativa and the structure of thymoquinone, the main component of $N$. sativa and many other plants 
Triterpenoids are one of the most abundant natural products in plants. They exhibit huge structural diversity as more than 90 different triterpenoidal carbon skeletons are known. Further oxidative modifications and glycosidation of the skeleton generate even more diversity [22]. Oleanolic (Figure 5a) and ursolic acids (Figure 5b) are among the well-known natural occurring pentacyclic triterpenoids that widely exist in many food products and in more than 120 plant species [23]. Oleanolic acid (OA) is known to possess anti-inflammatory, antitumor, antiviral, hepatoprotective and antihyperlipidemic effects. Moreover, it has been used in Chinese traditional medicine to treat liver disorders for over twenty years. Ursolic acid (UA) is also known to exhibit a wide and interesting biological activities including anti-inflammatory, anti-ulcer, antihyperlipidameic, antihyperglycaemic, hepatoprotective, neuroprotective and anticacinogenic activities [23]. The oral bioavailability of these two natural triterpenoidal acids is greatly limited by their very poor solubility in water. In fact, this drawback limits their development as a medicine as well as their use in food, health and cosmetic products.

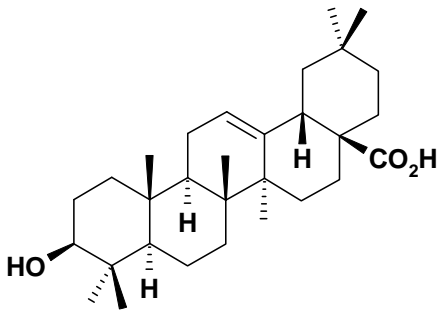

a

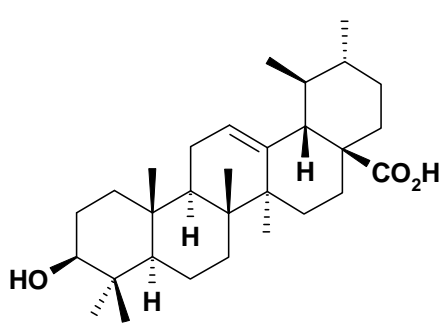

b

Figure 5. Structures of a) oleanolic acid and b) ursolic acid

Asiatic acid (Figure 6) is another natural derivative of oleanolic acid. This compound is known to be clinically effective on systemic scleroderma, abnormal scar formation and keloids [24]. Again, the poor solubility of this compound in water limits its bioavailability and hence hinders its usage as a drug.

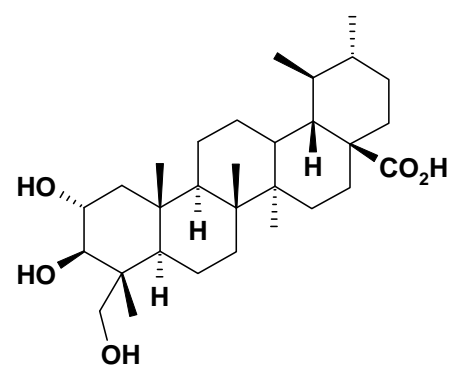

Figure 6. Structure of Asiatic acid 
Cucurbitacins (Figure 7) resemble another class of triterpenes with interesting pharmaceutical properties. Cucurbitacins are a group of highly oxidized tetracyclic triterpenoids that are widely distributed in the plant kingdom and well recognized for their bitterness and toxicity. Such compounds were initially isolated from plants belonging to the plant family Cucurbitaceae, but were later found to be present, either as non-glycosylated or glycosylated in many plant families including Brassicaceae, Scrophulariaceae, Begoniaceae, Elaeocarpaceae, Datiscaceae, Desfontainiaceae, Polemoniaceae, Primulaceae, Rubiaceae, Sterculiaceae, Rosaceae and Thymelaeaceae. In plants, cucurbitacins are known to act as heterologous chemical pheromones that protect plants from external biological insults [25]. Moreover, these compounds are known to possess a broad range of potent biological activity due to their cytotoxic properties. In traditional medicine, cucurbitacins-containing plants have been known for their antipyretic, analgesic, anti-inflammatory, antimicrobial, and antitumor activities [26].

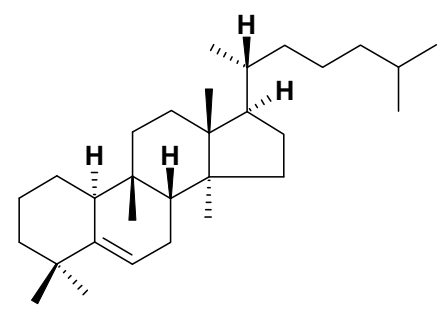

Figure 7. Basic structure of a cucurbitacin

There are 17 main molecules from cucurbitacin A to cucurbitacin T, and hundreds of compounds derived from them. Among them, cucurbitacins B, D, F, I and compounds derived from them have been extenively investigated for their anticancer activities.

\section{Alkaloids}

Alkaloids are a highly diverse class of secondary metabolites, with more than 5000 compounds being identified ranging from simple to highly complicated structures. These compounds contain a ring structure and a nitrogen atom, in most cases, the nitrogen is part of a heterocyclic ring structure. Alkaloids are known to exhibit significant biological activities. Examples include the relieving action of ephedrine for asthma, the analgesic action of morphine and the anticancer effects of vinblastine. Vinca alkaloids like vinblastine, vincristine, and vinorelbine are widely used cytotoxic drugs that elicit their effects through disruption of microtubules, resulting in metaphase arrest in dividing cells [27]. Thus, these compounds would benefit from a controlled release dosage form that would result in a prolonged duration of exposure over extended period of time. However, in spite of their significant bioactivities, these compounds suffer from side effects. The major adverse effect of vinblastine is hematologic toxicity which occurs much more frequently than with vincristine therapy. Other side effects include nausea, vomiting and constipation, dyspnea, chest or tumor pain, wheezing and fever. Many recent 
publications dealt with loading vinca alkaloids in liposomal nanocarriers to lessen such side effects $[27,28]$.

\section{Nanoflora}

As discussed above, herbal medicines are accompanied with many problems that prevent them from reaching their full potential as pharmaceutical formulations. These problems include but not limited to: low solubility in water, low bioavailability, high toxicity and instability. Nanotechnology has shown great promise for many medical applications such as cancer diagnosis, chemotherapeutic drug delivery, and diabetes treatments [29]. This technology is beneficial in overcoming some difficulties encountered with using bulk variable drug molecules in their synthetic and natural forms.

In the last decade, tremendous attentions have been paid on replacing synthetic drugs by natural bioactive phytochemicals to eliminate synthetic drugs side effects. In order to reach this goal, the above mentioned limitations need to be overcome. Nanotechnology can play an important role in reducing or even eliminating such drawbacks. Such possibility will open the door for a wide range of candidate compounds that were overlooked in the past due to these limitations, to be revisited again. In this chapter, a systematic overview of the various methods that can be applied to overcome one or more of these limitations and will lead finally to an acceptable formulation composed mainly of the active phytochemical attached to or encapsulated in a nanocarrier system forming what will be known throughout this chapter as a nanoflora. Such combination is capable of reaching the final phases of testing these active compounds and be helpful in improving health care systems.

\section{Nanotechnology and nanoparticles}

Several nanoparticle systems have been used to aid in the formulation, encapsulation and release of active compounds extracted or derived from natural resources. The main types of these particles are liposomes, solid lipid nanoparticles, inorganic nanoparticles, microemulsion, polymer nanoparticles, dendrimers and many other types.

\subsection{Micelles and Liposomes}

Micelles are spontaneous aggregates of amphiphiles (such as surfactants, Figure 8a) with usually spherical structures with a size range of 5-25 nm (Figure $8 b$ ). Their core is usually hydrophobic if they aggregate in polar media, however, they do form inverted micellar system in non polar media with a hydrophilic core. Micelles are perfect carriers for drugs and have been used more than any other system of nanoparticles [30]. Micelles come first in mind when tackling solubility issues in aqueous media [31,32]. The solubilization power of micelles has been known and used for a long time especially as detergent. Their mechanism of action was discovered in early 1900s. Micelles can be used as drug carriers and as solubilizing agents. 
However, they cannot be used to tackle other problems accompanying natural active compounds such as toxicity and stability since micellar systems are very dynamic ones and suffer from fast clearance rate and stability issues. However, some of these disadvantages were to some extent reduced via the preparation of polymeric micelles [33]. Polymeric micelles are more stable with longer shelf life and stay longer in the body.

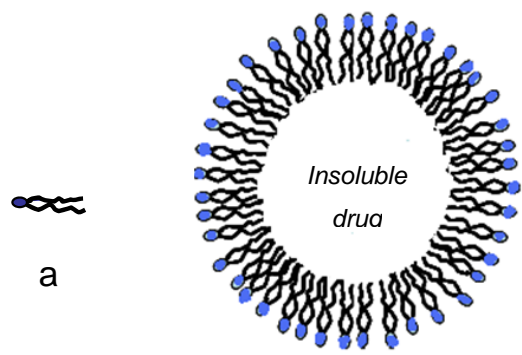

b

Figure 8. a) Sketch of a lipid or surfactant molecule capable of forming micelles and liposomes with a polar head and non-polar tail, b) Schematic presentation of a micelle with an insoluble drug encapsulated in the vicinity made by the non-polar tails

Liposomes are spherical vesicles that are composed of lipid bilayer (Figure 9a). Liposomes were discovered in 1961 by the British haematologist Alec Bangham and its resemblance to the cell membrane attracted immediate attention $[34,35,36]$. The name liposome was derived from the two Greek words lipo meaning fat and soma meaning body, which perfectly describes these spherical objects that are made mainly from lipids. In some cases other constituents are added to modify their chemical and physical properties (Figure 9b). Liposomes are easily prepared by disturbing the lipid film in aqueous medium. This disturbance may be a result from a large shear force produced via several techniques such as sonication. Liposomes are different from micelles (Figure 9) in that they are composed of bilayer lipid membrane whereas micelles are made from monolayer lipid vesicles.

There are different types of liposomes, including Small Unilamellar Vesicles (SUV, Figure 10b), Multilamellar Vesicles (MLV, Figure 10c), Large Unilamellar Vesicles (LUV, Figure 10d), Multivesicle Vesicles (MVV, Figure 10e) and cochleate vesicles (Figure 11). Each type of liposome is formed depending on experimental conditions. In addition, a dominant liposome type, size can be determined and/or made, after they are prepared via a series of extrusion process accompanied by shear or via several freeze-thaw processes since these structures are dynamic.

Liposomes are very important as drug carrier systems due to many factors including their suitability to encapsulate polar and non polar drugs, their stability and long shelf life, controllable properties such as size and charge, ability to functionalize and modify the surface due to the presence of many functional groups, and finally their biocompatibility and degradability. However, liposomes suffer from various disadvantages which include their short half-life 


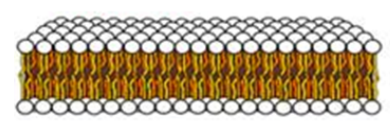

a

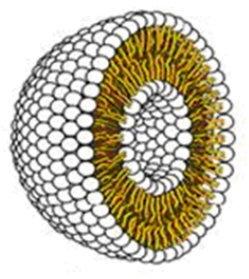

b

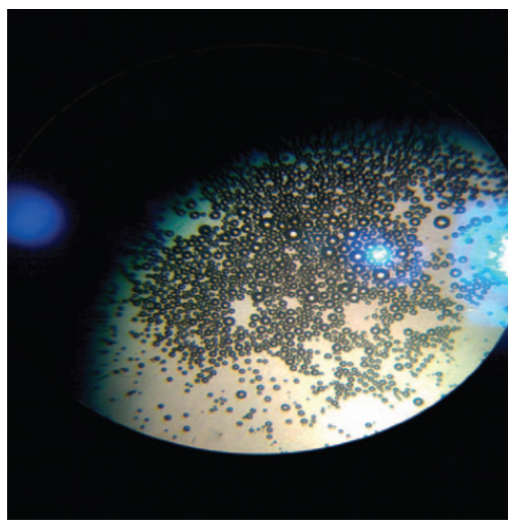

C

Figure 9. Basic structure of: a) bilayer lipid sheet, b) unilamellar liposome and c) optical micro-image of liposomes loaded with Thymoquinone [37]

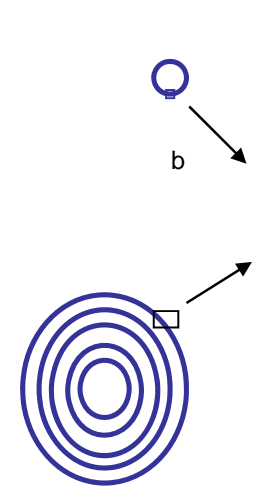

d

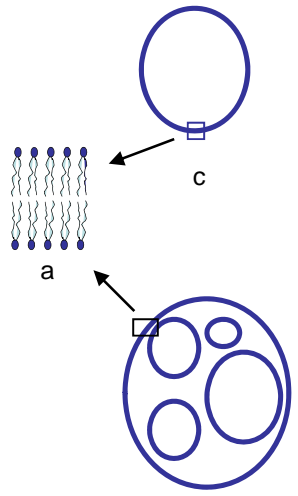

e

Figure 10. Schematic representation of the different types of liposomes a) the lipid bilayer, b) Small Unilamellar Vesicle (SUV), c) Large Unilamellar Vesicle (LUV), d) Multi Lamellar Vesicle (MLV) and e) Multi Vesicle Vesicle (MVV).

in the circulation system, although it can be enhanced by better controlling the size of the liposome vesicle and modifying its composition. Even though liposomes are suitable to encapsulate non polar drugs in the hydrophobic bilayer of the vesicle (Figure 9a and 10a), sometimes, such drugs affect the integrity of these vesicles rendering them unsuitable for non polar drugs.

Another promising type of nanoparticles is the phytosomes. The phospholipid in these types of nanoparticles (considered mainly as liposomes) is covalently attached to the phytochemical. Phytosomes are gaining increased interest and are the focus of more research to be used as drug delivery systems [38, 39, 40]. 


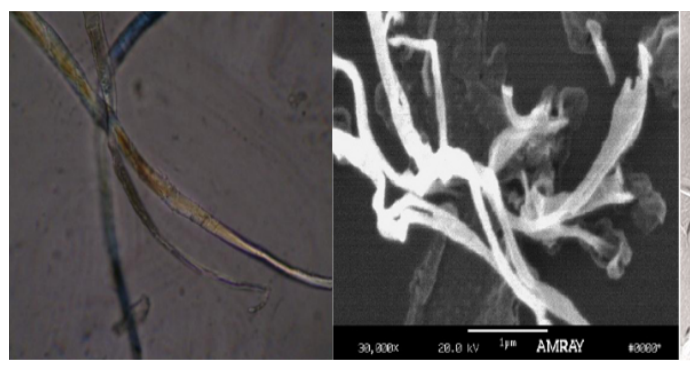

(a) (b)

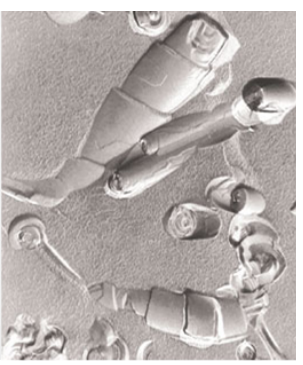

(c)

Figure 11. a) Optical micro image of cochleates [41], b) SEM image of cochleates [42], and c) freeze fracture electron micrographs of cochleates cylinders [42]

\subsection{Solid Lipid Nanoparticles (SLN)}

These are usually spherical structures composed of a lipid core, capable of solubilizing lipophilic drugs, surrounded with surfactants that stabilizes the lipid core and can be used for the hydrophilic drugs and other fictionalizations processes (Figure 12) [43]. SLN share other types of nano-carriers' their common advantages like their suitability to encapsulate non polar insoluble drugs in its polymeric core, shielding the drug the outside environment - which could be sometimes harsh - and as consequence increases the drug stability and reduces its toxicity to the body. Other advantages include not only the ability to functionalize the SLN surface with markers and targeting devices in order to enhance the targeting process, but also their ability to produce a sustained and slow release of the drug in the targeted site. However, SLN have advantages over other types of delivery systems in that they are easier to prepare, cheaper and much easier for scale up productions.

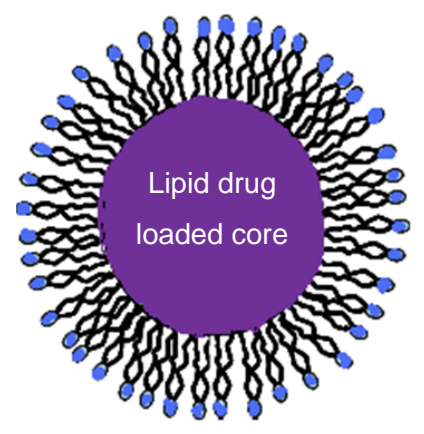

Figure 12. Structure of Solid Lipid Nanoparticle (SLN) stabilized with surfactant molecules 


\subsection{Polymer Nanoparticles}

Another type of particles that draw large attention is polymer nanoparticles [44]. These types of nanoparticles (Figure 13) are easily made-mostly from biodegradable polymers and can increase the stability and time of circulation. Moreover, in addition of being non toxic, other advantages of these polymer nanoparticles include controlled drug release, biocompatability and their suitability for scale up methods [45]. The most used two polymers include both poly (lactide-co-glycolic acid) (PLGA) and poly (lactic acid) (PLA) [46]. Other polymers are also good candidates to form polymeric nanoparticles and be suitable to act as drug carriers. These include sugars [47], proteins [48] such as albumin [49], gelatin nanoparticles [50] and many other naturally occurring macromolecules.

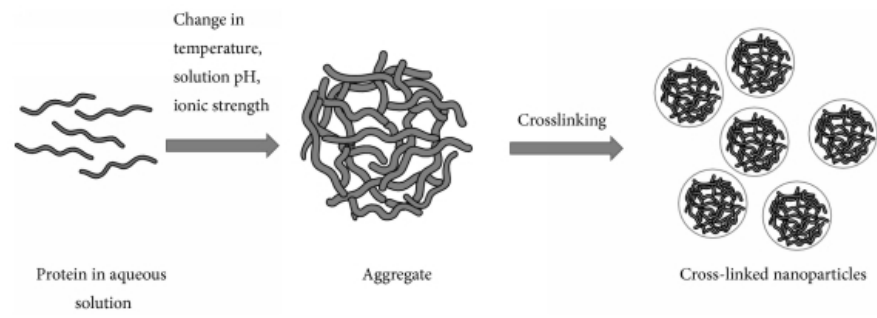

Figure 13. Schematic representation of polymer nanoparticles preparation [48]

Dendrimers are repetitive branched molecules attached to each other in a tree-like manner and typically are symmetric around their core. They can also be categorized as polymeric nanoparticles, and are characterized by their structural perfection, water solubility and monodispersity. Dendrimers are good encapsulating agents for hydrophobic drugs due to their non polar core.

\subsection{Microemulsions (ME)}

Microemulsions are usually made from oil, water, surfactant and a co-surfactant. They are thermodynamically stable (in contrast to emulsions), transparent, and form spontaneously. The particle size ranges from 10 to $100 \mathrm{~nm}$, which enhances their penetration through cellular membranes making microemulsions suitable as drug carriers. Due to the presence of polar and non polar components in ME's, they are very good solubilizing agents. Their properties can be adjusted to suit the drug to be carried by optimizing compositions, types of both the surfactant and the co-surfactant in addition of course to the oil used in the composition [51].

\subsection{Inorganic nanoparticles}

Inorganic nanoparticles such as gold nanoparticles, silver nanoparticles, ceramics, carbon nanoparticles and nanotubes were the focus of very extensive research and in many fields. Inorganic nanoparticles can be mainly classified into three different main categories including 
the: i) transition metal nanoparticles, ii) ceramics nanoparticles and iii) carbon nanoparticles in addition to other types.

Transition metal nanoparticles (Figure 14) such as $\mathrm{Au}, \mathrm{Ti}, \mathrm{Pt}$ are gaining increasing interest in the medical field [52]. There are many methods were transition metals can be applied in medicine, drug delivery being one of them. For example, many transition metals can act as drugs themselves when excited by light radiation. Depending on the excitation process and the type of metal involved, when the absorbed energy is released, it can damage the DNA and/ or modify the protein, promote lipid peroxidation and destroy the cell microenvironment, hence causing cell death. This method is promising in fighting cancer cells. Also, these nanoparticles can be very powerful in imaging, which is very important in both diagnosis and therapy monitoring [53]. One of the most promising nanoparticles in combating cancer is $\mathrm{Au}$ nanoparticle [54]. However, these metals can be used as drug carriers. For example, Au shuttles can be used for site specific delivery of toxic drugs [52]. However, these nanoparticles suffer from loaming safety and clearance concerns. Metals in the nanometer scale range have special properties and penetrations, making them very powerful catalysts that can trigger undesired reaction. Nowadays, there are large concerns regarding safety of specifically metal nanoparticles and nanoparticles in general.

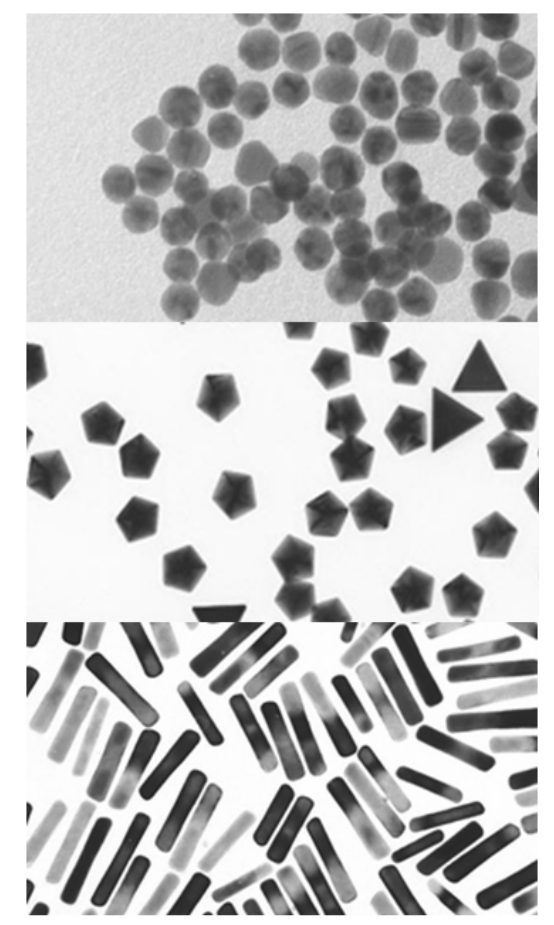

Figure 14. TEM images of metal nanoparticles with different geometries [55] 
Ceramics nanoparticles are mostly composed from oxides, nitrides and carbides with silica (Figure 15) $\left(\mathrm{SiO}_{2}\right)$ being the most used. Mainly they are used as hollow shells or cores that are coated with biodegradable and biocompatible polymers. Such surface modifications improve the properties of these nanoparticles especially for targeted delivery.
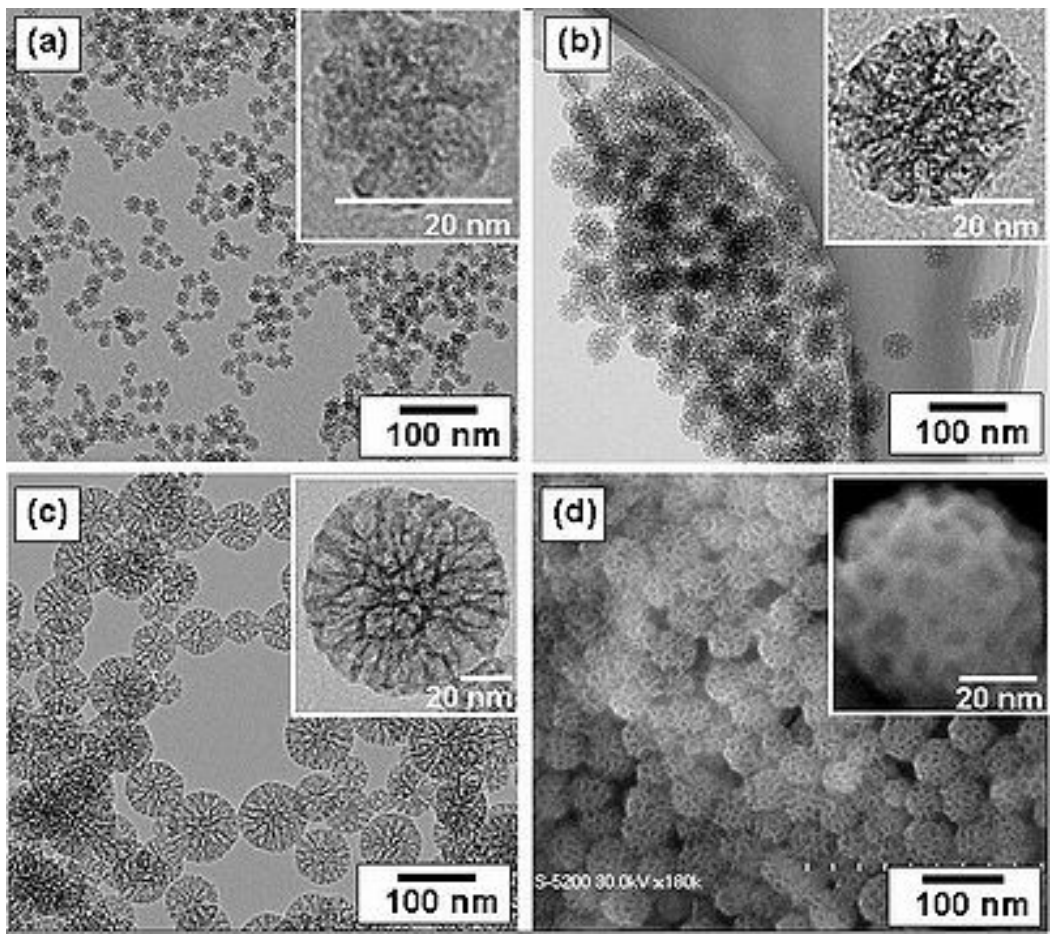

Figure 15. SEM images of silica nanoparticles with different sizes

\section{Enhancing solubility and bioavailability}

Low solubility of active phytochemicals is considered the main obstacle that hinders their usage in pharmaceutical formulations. This is due to two main reasons; the first is that the medium at which drugs work is mainly aqueous. Low solubility in aqueous media will drastically lower the concentration of the drug causing a poor bioavailability. Nanoparticles can provide an alternative medium for these drugs to be solubilized in and carried out through the body to the targeted tissue or organ. The extensively small sizes of nanoparticles give high surface area to volume ratio and as a result, more and more water molecules can surround the particles and the solubility of hydrophobic compound is enhanced [56]. 
Several nano-vesicles can be used to enhance the solubility such as micelles, liposomes, solid lipid nanoparticles, polymer nanoparticles and many others [57]. Triptolide, is an example of a bioactive diterpenoid epoxide ingredient isolated form Tripterygium wilfordii, a plant used in traditional Chinese medicine. This compound was found to be active in vivo and in vitro mouse models against polycystic kidney disease and against pancreatic cancer. It can also be used in the treatment of autoimmune diseases especially rheumatoid arthritis, psoriasis, and leukemia. However, it suffers from low solubility and high toxicity. In order to overcome its solubility and toxicity issues, it was prepared as a biocompatible and biodegradable tripolide-loaded poly [DL-lactic acid] nanoparticles [58]. It was also studied as a micro-emulsion system for transdermal delivery.

The solubility of quercetin (a flavonoid that is naturally present in a wide range of fruits and vegetables especially in onion, apples and many edible fruits) was enhanced 100 times after encapsulation in polymeric nanoparticles suspensions [59].

Tetrandrine, bis-benzylisoquinoline alkaloid, exhibits antitumor activity and is known to acts as a nonselective calcium channel blocker. This compound has very limited clinical applications due to its poor water solubility. However, the solubility of this alkaloid was enhanced as a result of its incorporation into SLN [60].

Cryptotanshinone is an active quinoid diterpene isolated from the roots of the Asian medicinal plant, Salvia miotiorrhiza Bunge. This diterpene is known to exhibit variable interesting pharmacological activities including anti-inflammatory, cytotoxic, anti-bacterial, anti-parasitic, anti-angiogenic and anti-oxidative activities but suffers from very low bioavailability as a result of its extremely low water solubility. Cryptotanshinone oral bioavailability was highly enhanced by introducing solid lipid nano-formulations [61].

Hypericin is a natural photosensitizer with limited ability to be used in diagnostic applications because of its high hydrophobicity and limited solubility. Different nano-formulations like hypericin-loaded solid lipid nanoparticles (Hy-SLN) and suspension of Hypericin-polymeric nanoparticles have been developed in order to obtain better photo-detection and photodynamic therapy $[57,62]$.

Thymoquinone (Figure 4) is an active ingredient found in Black Seeds (N. sativa). It has anticancer activity in addition to other therapeutic effects [13, 18, 63]. However, this compound suffers from poor solubility and high hydrophobicity leading to poor formulations for pharmaceutical applications. This problem was solved by encapsulating thymoquinone in various carriers such as polymer nanoparticles [45], liposomes (Figure 16) [37] and in cyclodextrin (Figure 17) [64].

Bioavailability can also be enhanced due to encapsulation of drugs or active compounds in nanocarriers. For example, ampelopsin, a flavonoid extracted from Ampelopsis grossedentata, is known to possess many pharmacological activities including anti-inflammatory, antimicrobial, anticarcinogenic activities in addition to its antioxidation, antihypertension, hepatoprotective and cough relieving effects. However, not only ampelopsin suffers from poor solubility in water, it also has very low permeability. Ampelopsin was successfully encapsulated in a 
microemulsion that enhanced its bioavailability by both enhancing its solubility and penetration through the intestinal mucosa [65].

(A)

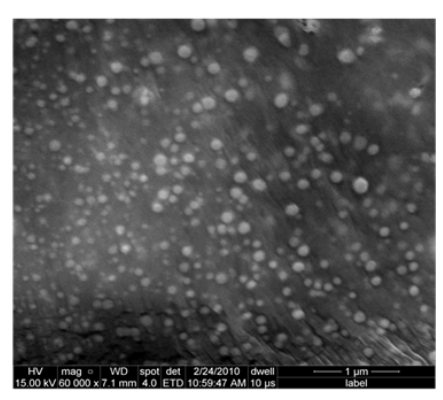

(B)

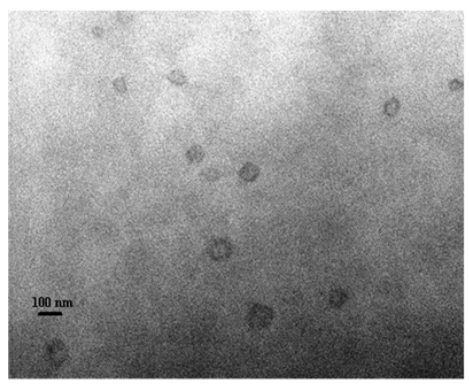

(C)

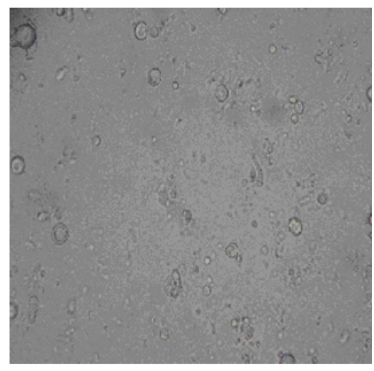

Figure 16. a) SEM, b) TEM and c) light microscope images of TQ-loaded liposomes [37]

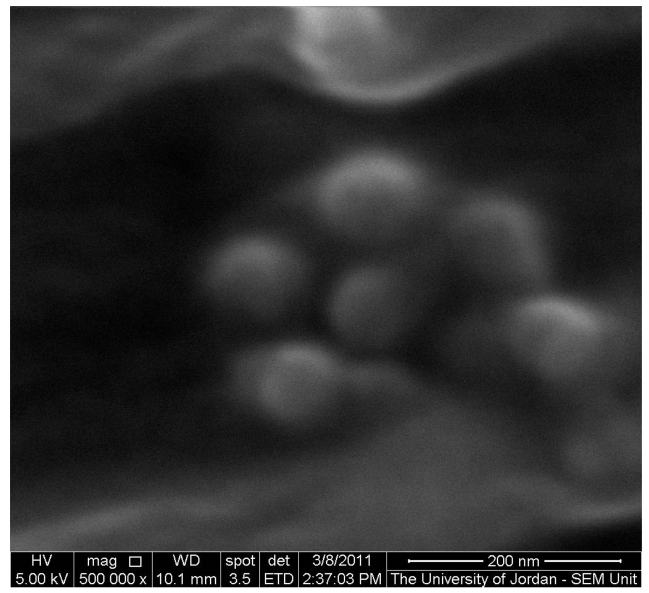

Figure 17. TEM image of a nanoparticle of thymoquinone with cyclodextrin $\beta$ [64]

\section{Reduced toxicity and side effects}

Most research focuses on reducing the toxicity and side effects of drugs, especially for those used in chemotherapy. Many Nanoparticles systems can be used to encapsulate the toxic drug and deliver it to specific sites in the body. These Nanoparticles are mainly made from biodegradable, biocompatible materials such as natural polymers which include polysaccharides 
and proteins. For example, triptolide, which was mentioned previously to have anti-tumor activity, has a side effect of being irritating to the gastric systems. Such gastric irritation can be reduced by changing the nanoparticle carrier. When triptolide is encapsulated in Solid Lipid Nanoparticles (SLN), such irritations are minimized [32]. Nanoparticles in general shield these toxic drugs and isolate them until reaching their destination via targeted delivery mechanism. Such mechanism of delivery depends on the capability to functionalize the surface of the nanoparticle carriers.

Targeted drug delivery is deliberately increasing the concentration of the drug in specific part of the body relative to other parts in order to increase the efficacy of drug and decrease the side effects. The complex cellular network of organisms makes targeting a difficult mission. There are variable methods of targeting such as modification of surface charge, inserting ligands, and using biomarkers. The targeting mechanism can be divided into two mechanisms: passive targeting and active targeting.

Passive targeting refers to the preferable accumulation of chemotherapeutic agent in solid tumor as a result of enhanced vascular permeability of tumor tissue as compared to healthy tissue [66].

Tumors have unique features, which make them distinct from normal tissue. The intact tissue has non leaky microvasculars, while tumor tissue has leaky capillary beds. This situation promotes the delivery and retention of drug loaded nonparticles through tumor tissue. This phenomenon is recognized as the enhanced permeability and retention effect (EPR). The hydrophobic surfaces of nanomaterials are highly susceptible to osponization and clearance $[66,67]$. When the surfaces were modified by becoming more hydrophilic, the rapid clearance problems can be solved and longer circulation can be obtained because the hydrophilic coating on the surfaces repels plasma and protein so drug loaded nanoparticles become invisible to mononuclear phagocytic system (MPS).

Active targeting refers to the use of drug carriers with ligands (antibody, peptide) that are selectively recognized by a receptor on the cell of interest. Since ligand-receptor interaction can be highly selective, a more precise targeting is achieved with improved target cell recognition and target cell uptake [67].

Particle size and size distribution are the most important characteristics of nanoparticle systems. Many studies have proved that nanometer particles are more effective and beneficial than micrometer particles in drug delivery system. In nano scale, the surface area to volume ratio is high; this situation makes the loaded drug less susceptible to reticular endothelial system clearances. The nano-sized particles have better ability to penetrate through cells and even small capillaries [68]. However, the ultimate small size of particles and large surface area lead sometimes to limited drug loading and burst release pattern [69]. Surface modification can be helpful in increasing residence time in the blood and reducing nonspecific distribution.Unsuccessful surface modification ultimately is the main limiting factor for long-circulating nanoparticle systems [70]. 


\section{Enhanced activity}

Encapsulation of active compounds into nanocarriers improves their activity. This might be attributed to several factors such as enhanced solubility, better stability for both in vivo and in vitro, and better formulation. However, the better penetration of the introduced nano systems through cellular membrane via unique interaction mechanism is an additional factor that participate in enhancing the bioactivity of drugs encapsulated in nanocarriers. For example, Origanum dictamnus extracts are known to have antimicrobial and antioxidant properties due to the presence of considerable concentrations of pehnolic compounds like flavones and cumarines. Research showed that encapsulating this extract into liposomes improved its activity [71].

\section{Release profile}

Obtaining constant drug release is considered an urgent necessity to obtain sustained drug level in tumor tissue which can lead to a lower dose requirement. For example, paclitaxel gelatin nanoparticles are highly effective in the treatment of bladder cancer because the rate of paclitaxel release from paclitaxel gelatin nanoparticles is limited by the drug solubility in aqueous medium. So the drug concentration remains constant and does not dilute when urine production is increased [58].

Sometimes, a fast release rate is desired, accompanied with enhanced solubility and penetration of non polar drugs. For example, Hibiscus rosa-sinensis and Murraya koenigii extracts are acclaimed to prevent hair loss and promoting its growth. However, proving such claims scientifically was hindered by many limitations, especially the poor solubility of the extracts in water. Since the desired formulation is intended to be used as a topical treatment with fast release rate to study its effectiveness, microemulsions were the ideal choice for improving the solubility and systemically study the extracts of these plants [72].

\section{Enhanced stability}

The instability of most phytochemicals in bio environments results in quick degradation and reduced activity. Encapsulation of such phytochemicals in carriers shields them from harsh conditions that lead to their decomposition. Quercetin has a life time of only five minutes in plasma whereas; quercetin loaded in liposomes modified with polyethylene glycol (PEG) has a life time of more than five hours in plasma [73]. Quercetin was also encapsulated in polymeric nanoparticles. Its antioxidant activity was maintained while its stability was highly enhanced when encapsulated in eudragit nanoparticles (polymeric nanoparticles). This polymeric nanoparticle protected quercetin and improved its stability in in acidic medium. However, the polymeric nanoparticles released the antioxidant under neutral to basic conditions, making 
such nanoparticles suitable for oral delivery of bioactive compounds and deliver them beyond the acidic stomach environment [74].

\section{Success stories}

Nanotechnology is one of the most powerful tools in the modern life, as it has made a revolutionary impact in every aspect of human life. The speed by which nanomedicine has advanced-particularly through the utilization of the various types of nanoparticles in the prevention, diagnosis and treatment of many complex diseases like cancer-is fascinating. The world is starting to witness the benefits of the application of nanotechnology in the field of herbal/natural products' drug delivery. One such benefit is Abraxane®. This drug is considered as a major success story of the nanomedicine approach to treat cancer [3]. Abraxane, an approved FDA drug, is a solvent free nano-version of the natural alkaloid, Taxol. Abraxane is both more effective and less toxic and has been successful in addressing the solubility problem associated with Paclitaxel (Taxol). Other examples of successful stories in the field of herbal drug delivery are the two companies Cosmetochem and Indena. Cosmetochem launched Herbasec ${ }^{\circledR}$ technology in the market. This product consists basically of liposomal encapsulated, standardized botanical extracts that are used in cosmetics for their antioxidant effects for the prevention of aging. Examples also include liposomal preparations of various herbal constituents like extracts of White tea, Green tea, white hibiscus, Gurana and Aloe vera. Indena commercializes the plant constituents/extracts of liquorice (18ß-glycyrrhetinic acid), Ammi visnaga (visnadin), Centella asiatica (triterpenes), Ginkgo biloba (ginkgoflavonglucosides, ginkgolides, bilobalide), Hawthorn flower (vitexin-2"-O-rhamnoside), milk thistle (silymarin and Silybin), horse chestnut (escin B-sitosterol), Terminalia sericea (sericoside), Panax ginseng (ginsenosides), grape seed (polyphenols) and Green tea (polyphenols) [75]. Moreover, a long list of recent patents on controlled release novel herbal formulations is becoming even longer [76] proving that nanotechnology for drug delivery is becoming the future of phytochemicals and opening the era for re-exploring and investigation the full potential power of traditional herbal medicine represented either by the herbal extracts and/or their pure isolated phytochemicals.

\section{Conclusions}

The application of nanotechnology to drug delivery has already had a significant impact on many areas of medicine. Currently, more than 20 nanoparticle therapeutics are in clinical use, validating the ability of nanoparticles to improve the therapeutic index of drugs. In addition to the already approved nanoparticles, numerous other nanoparticle platforms are currently under various stages of preclinical and clinical development, including various liposomes, polymeric micelles, dendrimers, quantum dots, gold nanoparticles, and ceramic nanoparticles. More complex systems such as multifunctional nanoparticles that are concurrently capable of targeting, imaging, and therapy are the subjects of future researches. 
Nanotechnology is offering several advantages to phytochemicals delivery and to drug delivery in general [77]. Of the many advantages, the most important ones are:

- Enhancing solubility and bioavailability.

- Reducing toxicity and side effects of these phytochemicals, especially that most bioactive phytochemicals are very toxic.

- Increasing the stability of active phytochemicals to stand harsh conditions along their way to their site of action.

- Enhancing the biocompatibility and reducing the toxicity of the formulation itself, since these nanocarriers limit the use of toxic non polar solvents that were traditionally used to increase solubility and improve the formulation characteristics.

- However, an important thing that we must pay attention to, is the safety and hazards of nanoparticles. In order to comprehend the safety of any formulation, long studies over a long period of time is needed. Nanoparticles are relatively new in the medical market and sector, and it is known that material in the nano scale usually has different properties compared with bulk scale, making it difficult to predict hazards of such small particles.

The currently approved nanoparticle systems have in some cases improved the therapeutic index of drugs by reducing drug toxicity or enhancing drug efficacy. Future research efforts need to be directed towards finding new methods for nanotoxicology, recognition of biological effects of nanoparticles in the environment, and creation of the bases of nanobiomonitoring.

\section{Author details}

Fadwa Odeh ${ }^{1}$, Hala Al-Jaber ${ }^{2}$ and Dima Khater ${ }^{1}$

*Address all correspondence to: f.odeh@ju.edu.jo

1 Department of Chemistry/The University of Jordan, Amman, Jordan

2 Department of Applied Sciences/Faculty of Engineering Technology/Al-Balqa Applied University/Marka, Amman, Jordan

\section{References}

[1] Matijevic E., editor. Fine Particles in Medicine and Pharmacy. New York: Springer; 2012. 
[2] Bonifacio BV, Bento da silva P, Aparecido M, Ramos S, Negri K M, Bauab TM and Chorilli M. Nanotechnology-based drug delivery systems and herbal medicines: A review, Inter J Nanomedicine, 2014, 9, 1-15.

[3] Bharali D J, Siddiqui I A, Adhami V M, Chamcheu J C, Aldahmash A M, Mukhtar H and Mousa S A. Nanoparticle Delivery of Natural Products in the Prevention and Treatment of Cancers: Current Status and Future Prospects. Cancers, 2011, 3, 4024-4045.

[4] Surangi H, Thilakarathna and Rupasinghe H P V. Flavonoid Bioavailability and Attempts for Bioavailability Enhancement, Nutrients, 2013, 5, 3367-3387.

[5] Mann J, Davidson R S, Hobbs J B, Banthorpe D V and Harborne J B. Natural Products: their Chemistry and Biological Significance, first edition 1994, Longman Group UK Limited.

[6] Munin A and Edwards-Lévy F. Encapsulation of Natural Polyphenolic Compounds; A Review. Pharmaceutics, 2011, 3, 793-829.

[7] Mignet N, Seguin J and Chabot G G. Bioavailability of Polyphenol Liposomes: A Challenge Ahead. Pharmaceutics, 2013, 5, 457-471.

[8] Martens S and Mithöfer A. Flavones and Favone Synthases, Phytochemistry, 2005, 66, $2399-2407$.

[9] Agrawal, A D. Pharmacological Activities of Flavonoids: A review, Int J Pharm Sci Nanotech., 2011, 4(2), 1394-1398.

[10] Sandhar H K, Kumar B, Prasher S, Tiwari P, Salhan M and Sharma P. A Review of Phytochemistry and Pharmacology of Flavonoids, Internationale Pharmaceutica Sciencia, 2011, 1(1), 25-41.

[11] Mori A, Nishino C, Enoki N and Tawata S. Cytotoxicity of Plant Flavonoids Against HeLa Cells, Phytochemistry, 1988, 27(4), 1017-1020.

[12] Bruschi M, Orlandi O, Rindone M, Rindone B, Saliu F, Suarez-Bertoa R, Tollpa E Zoia L. Podophyllotoxin and Antitumor Synthetic Aryltetralines. Toward a Biomimetic Preparation. In: Mukherjee A. (ed.) Biomimetics Learning from Nature, InTech, 2010, Available from http://www.intechopen.com/books/biomimetics-learning-from-nature/podophyllotoxin-and-antitumor-synthetic-aryltetralines-toward-a-biomimeticpreparation.

[13] Gali-Muhtasib H, Diab-Assaf M, Boltze C, Al-Hmaira J, Hartig R, Roessner A and Schneider-Stock R. Thymoquinone Extracted from Black Seed Triggers Apoptotic Cell Death in Human Colorectal Cancer Cells via a P53-Dependent Mechanism, Int J Oncol., 2004, 25(4):857-66. 
[14] Worthen D R, Ghosheh O A and Crooks P A. The in vitro Anti-Tumor Activity of Some Crude and Purified Components of Black Seed Nigella sativa L., Anticancer Research, 1998, 18, 1527-1532.

[15] Thoppil R J and Bishayee A. Terpenoids as Potential Chemopreventive and Therapeutic Agents in Liver Cancer, World J Hepatol, 2011, 3(9): 228-249.

[16] Rabi T and Bishayee A. Terpenoids and Breast Cancer Chemoprevention, Breast Cancer Res and Treatment, 2009, 115(2), 223-239.

[17] Kaseb A O, Chinnakannu K, Chen D, Sivanandam A, Tejwani S, Menon M, Dou Q P and Prem-Veer Reddy G. Androgen Receptor- and E2F-1-Targeted Thymoquinone Therapy for Hormone-Refractory Prostate Cancer, Cancer Res., 2007, 67(16):7782-7788.

[18] Mansour M A, Nagi M N, El-Khatib A S and Al-Bekairi A M. Effects of Thymoquinone on Antioxidant Enzyme Activities, Lipid Peroxidation and DT-Diaphorase in Different Tissues of Mice: A Possible Mechanism of Action, Cell Biochem Funct., 2002, 20(2), 143-151.

[19] Chehl N, Chipitsyna G, Gong Q, Yeo C J and Arafat H A. Anti-inflammatory Effects of the Nigella sativa Seed Extract, Thymoquinone, in Pancreatic Cancer Cells, HPB (Oxford), 2009, 11(5), 373-381.

[20] El Mahdy M, Zhu Q, Wang Q, Wani G and Wani A. Thymoquinone Induces Apoptosis through Activation of Caspase- 8 and Mitochondrial Events in p53-null Myeloblastic Leukemia HL-60 Cells, Int J Cancer, 2005, 117, 409-417.

[21] Shoieb A M, Elgayyar M, Dudrick P S, Bell J L and Tithof P K. In vitro Inhibition of Growth and Induction of Apoptosis in Cancer Cell Lines by Thymoquinone, International Journal of Oncology, 2003, 22, 107-113.

[22] Kushiro T, Shibuya M, Masuda K and Ebizuka Y. Mutational Studies on Triterpene Synthases: Engineering Lupeol Synthase into $\beta$-Amyrin Synthase, J. Am. Chem. Soc., 2000, 122, 6816-6824.

[23] Yang L, Sun Z, Zu Y, Zhao C, Sun X, Zhang Z and Zhang L. Physicochemical Properties and Oral Bioavailability of Ursolic acid Nanoparticles using Supercritical Antisolvent (SAS) Process. Food Chemistry, 2012, 132, 319-325.

[24] Songbai L, Qiang M A, Gaihong A N, NA Zhang N A, Jing W, Chuanxiang X, Bo C, Xiaojun S, Ron F, Yongcong S and Xuewei C. Preparation and Evaluation of Solid Dispersion of Asiatic Acid with PVPK30, Digest J of Nanomaterials and Biostructures, 2013, 8(2), $685-690$.

[25] Alghasham A A. Cucurbitacins - A Promising Target for Cancer Therapy, International Journal of Health Sciences, Qassim University, 2013 (Safar 1434H), 7(1). 
[26] Lee D H, Iwanski G B and Thoennissen N H. Cucurbitacin: Ancient Compound Shedding New Light on Cancer Treatment, The Scientific World Journal, 2010, 10, 413-418.

[27] Noble C O, Guo Z, Hayes M E, Marks J D, Park J W, Benz C C, Kirpotin D B and Drummond D C. Characterization of Highly Stable Liposomal and Immunoliposomal Formulations of Vincristine and Vinblastine, Cancer Chemother Pharmacol., 2009, 64, 741-751.

[28] Zhigaltsev I V,Maurer T N, Akhong Q, Leone R, Leng E, Wang J, Semple S C and Cullis P R. Liposome-Encapsulated Vincristine, Vinblastine and Vinorelbine: A Comparative Study of Drug Loading and Retention, Int. J. Mol. Sci., 2012, 13, 12598-12607.

[29] Melancon M P, Stafford R J and Li C. Challenges to effective cancer nanotheranostics, J. Control. Release, 2012, 164, 177-182.

[30] Husseini G and Pitt W. Micelles and Nanoparticles for Ultrasonic Drug and Gene Delivery, Adv Drug Deliv Rev., 2008, 60(10), 1137-1152.

[31] Kim S, Shi Y, Kim J, Park K and Cheng J. Overcoming the Barriers in Micellar Drug Delivery: Loading Efficiency, in vivo Stability, and Micelle-Cell Interaction, Expert Opin. Drug Deliv., 2010, 7(1), 49-62.

[32] Zhang N, Wardwell P R and Bader R A. Polysaccharide-Based Micelles for Drug Delivery, Pharmaceutics, 2013, 5, 329-352

[33] Francis M, Cristea M and Winnik F. Polymeric Micelles for Oral Drug Delivery: Why and How, Pure Appl. Chem., 2004, 76(7-8), 1321-1335.

[34] Bangham A D, Horne R W, Glauert A M, Dingle J T and Lucy J A. Action of Saponin on Biological Cell Membranes, Nature, 1962, 196, 952-955.

[35] Horne R W, Bangham A D and Whittaker V P. Negatively Stained Lipoprotein Membranes, Nature, 1963, 200, 1340.

[36] Bangham A D and Horne R W. Negative Staining of Phospholipids and Their Structural Modification by Surface-Active Agents As Observed in the Electron Microscope, Journal of Molecular Biology, 1964, 8, 660-668.

[37] Odeh F, Ismail S I, Abu-Dahab R, Mahmoud I S and Al Bawab A. Thymoquinone in Liposomes: A Study of Loading Efficiency and Biological Activity Towards Breast Cancer, Drug Delivery, 2012, 19(18), 371-377.

[38] Patel J, Patel R, Khambholja K and Patel N. An Overview of Phytosomes as an Advanced Herbal Drug Delivery System, Asian J Pharm Sci., 2009, 4(6), 363-371.

[39] Jain N, Gupta B, Thakur N, Jain R, Banweer J, Jain D and Jain S. Phytosome: A Novel Drug Delivery System for Herbal Medicine, Int. J. Pharm. Sci Drug Research, 2010, 2(4), 224-228. 
[40] Kareparamban J, Nikam P, Jadhav A and Kadam V. Phytosome: A Novel Revolution in Herbal Drugs, Int J Research Pharm Chem., 2012, 2(2), 299-310.

[41] Landge A, Pawar A and Shaikh K. Investigation of Cochleates as Carriers for Topical Drug Delivery, Int. J. Pharm. Pharm. Sci., 2013, 5(2), 314-320.

[42] Ramasamy T, Khandasamy U, Hinabindhu R and Kona K. Nanocochleate - A New Drug Delivery System, FABAD J. Pharm. Sci., 2009, 34, 91-101.

[43] Üner M and Yeren G. Importance of Solid Lipid Nanoparticles (SLN) in Various Administration Routes and Future Perspectives, In. J Nanomedicine, 2007, 2(3), 289-300.

[44] Soppimatha K, Aminabhavia T, Kulkarnia A and Rudzinski W. Biodegradable Polymeric Nanoparticles as Drug Delivery Devices, 2001, 70, 1-20.

[45] Ravindran J, Nair H B, Sung B, Prasad S, Tekmal R R and Aggarwal B B. Thymoquinone Poly(lactide-co-glycolide) Nanoparticles Exhibit Enhanced Antiproliferative, Anti-inflammatory, and Chemosensitization Potential, Biochem Pharmacol., 2010, 79,1640-1647.

[46] Abdollahi and Lotfipour. PLGA-and PLA-based Polymeric Nanoparticles for Antimicrobial Drug Delivery, Biomed Int., 2012, 3, 1-11.

[47] Kim I S and Kim S H. Development of a Polymeric Nanoparticulate Drug Delivery System in vitro Characterization of Nanoparticles Based on Sugar Containing Conjugates, Int J Pharm., 2002, 245, 67-73.

[48] Lohcharoenkal W, Wang L, Chen Y and Rojanasakul Y. Protein Nanoparticles as Drug Delivery Carriers for Cancer Therapy, Biomed Research International, 2014, 1-13.

[49] Kratz F, Fichtner I and Beyer U. Antitumor Activity of Acid Labile Tansferrin and Albumin Doxorubicin Conjugates in in vitro and in vivo Human Tumour Xenograft Model, European Journal of Cancer, 1997, 33, 175-175.

[50] Ziv O, Avtalion R and Margel S. Immunogenicity of Bioactive Magnetic Nanoparticles: Natural and Acquired Antibodies, Journal of Biomedical Materials Research A, 2008, 85(4)1011-1021.

[51] Muzaffar F, Singh U K and Chauhan L. Review on Microemulsion as Futuristic Drug Delivery, Int J Pharm Pharm Sci., 2013, 5(3), 39-53.

[52] Bhattacharyya S, Kudgus R, Bhattacharya R and Mukherjee P. Inorganic Nanoparticles in Cancer Therapy, Pharm Res., 2011, 28(2), 237-259.

[53] Liong M, Lu J, Kovochich M, Xia T, Ruehm S, Nel A, Tamanoi F and Zink J. Multifunctional Inorganic Nanoparticles for Imaging, Targeting, and Drug Delivery, ACS Nano., 2008, 2(5), 889-896.

[54] Cai W, Gao T, Hong H and Sun J. Applications of Gold Nanoparticles in Cancer Nanotechnology, Nanotechnology, 2008, 1, 17-32. 
[55] Lakshminarayana P and Liz-Marzan L. Growth and Galvanic Replacement of Silver Nanocubes in Organic Media, Nanoscale, 2013, 5, 4355-4361.

[56] Huang C. Studies on Phosphatidylcholine Vesicles, Formation and Physical Characteristics, Biochemistry, 1969, 8, 344-352.

[57] Thapa R, Khan G, Parajuli-Baral K and Thapa P. Herbal Medicine Incorporated Nanoparticles: Advancements in Herbal Treatment, Asian J Pharma Clin Res., 2013, 3(24), 7-14.

[58] Zhang C, Gu C, Peng F, Liu W, Wan J, Xu H, Lam C W and Yang X. Preparation and Optimization of Triptolide-loaded Solid Lipid Nanoparticles for Oral Delivery with Reduced Gastric Irritation, molecules, 2013, 18, 13340-13356.

[59] Yen F L, Wu T H, Lin L T, Cham T M and Lin C C. Nanoparticles Formulation of Cuscuta chinensis Prevents Acetaminophen-Induced Hepatotoxicity in Rats, Food Chem Toxicol., 2008, 46, 1771-1777.

[60] Li Y, Dong L, Jia A, Chang X and Xue H. Preparation and Characterization of Solid Lipid Nanoparticles Loaded Traditional Chinese Medicine, Int J Biol Macromolecules, 2006, 38, 296-299.

[61] Hu L D, Xing Q, Meng J and Shang C. Preparation and Enhanced Bioavailability of Cryptotanshinone-Loaded Solid Lipid Nanoparticles, AAPS PharmSciTech., 2010, $11(2), 582-587$.

[62] Limaa A, Pizzolb C, Monteirob F, Creczynski-Pasab T, Andradec G, Ribeiroc A and Perussia J. Hypericin Encapsulated in Solid Lipid Nanoparticles: Phototoxicity and Photodynamic Efficiency, J of Photochemistry and Photobiology B: Biology, 2013, 125(1) 146-154.

[63] Zihlif M A, Mahmoud I S, Ghanim M T, Zreikat M S, Alrabadi N, Imraish A, Odeh F, Abbas M A and Ismail S O. Thymoquinone Efficiently Inhibits the Survival of EBVInfected B Cells and Alters EBV Gene Expression, Integr. Cancer Ther., 2013, 12(3), 257-263.

[64] Abu Dahab R, Odeh F, Ismail S I, Azzam H and Al Bawab A. Preparation, Characterization and Antiproliferative Activity of Thymoquinon- $\beta$-cyclodextrin Self Assembly Nanoparticles, Die Pharmazie, 2013, 68, 939-944.

[65] Solanki S S, Sarkar P and Dhanwani R K. Microemulsion drug delivery system: for bioavailability enhancement of ampelopsin, ISRN Pharmaceutics, 2012, Article ID 108164, 4 pages, doi:10.5402/2012/108164.

[66] Lammers T, Kiessling F, Hennink. W and Storm G. Drug Targeting to Tumors: Principles, Pitfalls and (Pre-) Clinical Progress, J Controlled Release, 2012, 161, 175-187.

[67] Caffo M, Raudino G and Caruso G. Nanotechnology and Brain Tumors Drug Delivery, Recent Patents on Nanomedicine, 2013, 3, 26-36. 
[68] Jahanshahi M and Babaei Z. Protein Nanoparticle: A Unique System as Drug Delivery Vehicles, African Journal of Biotechnology, 2008, 7 (25), 4926-4934.

[69] Singh A, Garg G and Sharma P. Nanospheres: A Novel Approach for Targeted Drug Delivery System, Int J Pharma Sci Review and Research, 2010, 5(3), 84-88.

[70] Alexi. F, Pridgen E and Farokhzad O. Factors Affecting the Clearance and Biodistribution of Polymeric Nanoparticles, molecular pharmaceutics, 2008, 4(5), 505-515.

[71] Gortzi O, Lalas S, Chinou I and Tsaknis J. Evaluation of the Antimicrobial and Antioxidant Activities of Origanum dictamnus Extracts Before and After Encapsulation in Liposomes, molecules, 2007, 12, 932-945.

[72] Kurup N S and Joshi P R. Formulation and Evaluation of Herbal Microemulsion for Controlling Hair Loss, Int J Res Pharm Sci., 2013, 4(3), 420-436.

[73] Yuan Z, Chen L, Fan L, Tang M, Yang G, Yang H, Du X, Wang G, Yao W,Zhao Q, Ye B, Wang R, Diao P, Zhang W, Wu H, Zhao X and Wei Y. Liposomal Quercetin Efficiently Suppresses Growth of Solid Tumors in Murine Models, Clin Cancer Res., 2006, 12(10), 3193-3199.

[74] Pool H, Quintanar D, Figueroa J, Figueroa, Bechara E, McClements D and Mendoza S. Polymeric Nanoparticles as Oral Delivery Systems for Encapsulation and Release of Polyphenolic Compounds: Impact on Quercetin Antioxidant Activity and Bioaccessibility, Food Biophysics, 2012, 7, 276-288.

[75] Verma H, Prasad S B, Yashwant and Singh H. Herbal Drug Delivery System: A Modern Era Prospective, Int J of Current Pharma Review and Research, 2013, 4(3), 88-101.

[76] Goyal A, Kumar S, Nagpal M, Singh I and Arora S. Potential of Novel Drug Delivery Systems for Herbal Drugs, Ind J Pharm Edu Res., 2011, 45(3), 225-235.

[77] De Jong W H and Borm P J A. Drug Delivery and Nanoparticles: Applications and Hazards, Int J Nanomedicine, 2008, 3(2), 133-149. 